\section{SEVERITY OF PAIN AT ARRIVAL AND MANAGEMENT OF PAINFUL SICKLE CELL CRISIS IN A FRENCH PEDIATRIC EMERGENCY DEPARTMENT}

L. Tostivint ${ }^{1}$, L. Al Lawati ${ }^{1}$, E. Courtois ${ }^{1}$, E. Lesprit ${ }^{2}$, B. Quinet ${ }^{2}$, J.-B. Armengaud ${ }^{1}$, M. Agogué ${ }^{1}$, R. Carbajal ${ }^{1}$

${ }^{1}$ Pediatric Emergency Department, Armand Trousseau University Hospital, AP-HP, ${ }^{2}$ General Pediatrics Department, Armand Trousseau University Hospital, AP-HP, Paris, France

Objectives: To describe (1) the severity of painful sickle cell crisis (PSCC) in children presenting to the ED and (2) the ED analgesic management.

Methods: Retrospective study of all one-year visits to an ED for a PSCC.

Results: From May 2008 to April 2009, 189 visits concerning 87 children were registered. In 149 $(79 \%)$ visits, an initial pain score was recorded. Assessments were carried out with four different scales. A 0-10 Visual Analog Scale was used in $97(65.1 \%)$ of assessments; 3 other scales were used for the remaining $52(34.9 \%)$ assessments. In order to uniform all the assessment data, the latter were transformed into a 0-10 scale. The median (interquartile) pain score of all assessments was 6 (4-8). The 149 pain assessments were divided into three groups: mild pain ( $\leq 4 / 10$ score) including $40(26.8 \%)$ assessments, moderate pain (5-7/10) including $61(40.9 \%)$ assessments and severe pain $(\geq 8 / 10)$ including $48(32.2 \%)$ assessments. The frequency of use of step $1 \mathrm{WHO}$ analgesics (acetaminophen, ibuprofen), step 2/a codeine, step $2 / b$ nalbuphine, and step 3 morphine were, respectively, $60 \%, 7.5 \%, 75 \%$, and $0 \%$ for mild pain, $60.7 \%, 8.2 \%, 73.8 \%$, and $1.6 \%$ for moderate pain, and $52.1 \%, 2.1 \%, 60,4 \%$ and $8.3 \%$ for severe pain. Nitrous oxide/oxygen was used for all venipunctures and in $38.3 \%$ of visits to alleviate persistent PSCC. In 124 of 149 (83\%) visits, children were admitted to the hospital.

Conclusion: In $1 / 3^{\text {rd }}$ of ED visits for PSCC, children were in severe pain. Morphine was rarely used even for severe pain.

\section{MANAGEMENT OF SEDATION, ANALGESIA, AND MUSCLE RELAXATION IN CRITICALLY ILL CHILDREN: A SURVEY IN PAEDIATRIC INTENSIVE CARE UNITS IN SPAIN}

\section{S. Mencía, M. Botrán, J. López-Herce, A. García, R. González, T. Gema, A. Carrillo \\ Pediatric Critical Care Unit, Gregorio Marañon Hospital, Madrid, Spain}

Introduction: To define the strategies for the use and monitoring of sedative, analgesic, and muscle relaxant medication in Spanish paediatric intensive care units (PICU).

Methods: A questionnaire with 102 questions was sent by e-mail to all Spanish PICUs.

Results: Replies were received from 36 of the 45 PICUs (80\%). A written protocol for sedation and analgesia was used in $64 \%$; this medication was adjusted according to the diagnosis and clinical status of the patient in $30 \%$ of the units. Midazolam was the most widely used drug for sedation, followed by ketamine and propofol. Fentanyl was the most widely used drug for analgesia, followed by paracetamol and metamizole. The combination of midazolam and fentanyl in continuous infusion was used most frequently in patients on mechanical ventilation (MV), followed by propofol. Scales to monitor sedation and analgesia were employed in $45 \%$ of PICUs, most used the Ramsay scale. BIS was used in $50 \%$ of PICUs. Muscle relaxants were administered to $26 \%$ of patients on MV; the most common indications for MV were head injury and severe respiratory disease. The principal methods for avoiding withdrawal syndrome were a progressive withdrawal of the drugs and morphine chloride.

Conclusions: Although there is insufficient scientific evidence to determine the ideal drugs for sedation and analgesia in the critically ill child and the methods for monitoring and control, the production of guidelines and written treatment and monitoring protocols could help to improve the management and control of sedation and analgesia. 